Research Article

Han Yanqing*, Dong Cheng, Xu Ling

\title{
Serum CA72-4 as a biomarker in the diagnosis of colorectal cancer: A meta-analysis
}

https://doi.org/10.1515/med-2018-0026

received December 19, 2017; accepted March 14, 2018

Abstract: The purpose of this meta-analysis was to investigate the serum CA72-4 as a biomarker in the diagnosis of colorectal cancer by pooling the open published data. Methods. An electronic search of databases Pubmed, Medline, Web of Science, Cochrane Embase CBM, and CNKI were performed by two reviewers (Han Yanqing, Dong Cheng) independently to identify the studies relevant to serum CA72-4 as a biomarker in the diagnosis of colorectal cancer. The patient number of true positive(tp), false positive(fp), false negative(fn) and true negative(tn) were extracted from each included study. The diagnostic performance of serum CA72-4 as a biomarker in the diagnosis of colorectal cancer was assessed by pooled sensitivity, specificity and hierarchical summary receiver operating characteristic curve (HSROC). All the data was pooled by MetaDiSc 1.4 and Stata/SE 11.0 statistical software. Results A total of 22 studies with 2474 colorectal patients and 1576 controls were included in the present study and meta-analysis. The combined diagnostic sensitivity and specificity were 0.50 (95\%CI:0.48-0.52) and 0.86 (95\%CI:0.84-0.88) for serum CA72-4 as a biomarker in the diagnosis of colorectal cancer. The pooled positive and negative likelihood ratio were 3.41(95\%CI:2.57-4.53) and $0.62(0.55-0.71)$. The pooled area under the ROC curve (AUC) was 0.73. Deeks'funnel plot and Egger's line regression test $(p=0.49)$ showed no significant publication bias in the present meta-analysis. Conclusion Due toits low diagnostic sensitivity, the diagnostic performance of serum CA72-4 as a biomarker for colorectal cancer screening is limited.

*Corresponding author: Han Yanqing, Department of Surgery ,Tianjin Huanhu Hospital, Tianjin Key Laboratory of Cerebral Vascular and Neurodegenerative Diseases ,Tianjin 300350,China, No. 6 Jizhao Road, Jinnan District Tianjin, 300350 PR China., Tel:+86-02259065336, E-mail:telann22@163.com

Dong Cheng, Xu Ling, Department of Surgery, TianjinHuanhu Hospital, Tianjin Key Laboratory of Cerebral Vascular and Neurodegenerative Disease ,Tianjin 300350, China
Keywords: Colorectal cancer; CA72-4; Biomarker; Diagnosis; Meta-analysis

\section{Introduction}

Colorectal cancer(CRC) is one of the most diagnosed malignant carcinoma in the digestive system [1]. In the United States, it was the $4^{\text {th }}$ most diagnosed cancer with an estimate of more than 140,000 new cases and 50,000 deaths in year 2012 [2]. However, the mortality from colorectal cancer has decreased by about 35\% in recent decades attributing the success to effective colorectal screening methods and treatment modalities. The occurrence and development of colorectal cancer is accompanied by a series of molecular biological changes. The ideal tumor marker for screening colorectal cancer should have high sensitivity and specificity with easy management.

CA72-4 is a glycoprotein found on the surface of many malignant carcinoma cells [3], including colorectal cancer, ovarian cancer [4], gastric cancer [5] and pancreatic cancer. Several published studies have evaluated the diagnostic performance of serum CA72-4 as biomarker for colorectal cancer diagnosis. In our present study, we included the previously published relevant studies about CA72-4 as a biomarker for colorectal diagnosis and pooled the data in order to further evaluate its clinical value.

\section{Materials and methods}

\subsection{Publication identification}

In order to identify all the relevant studies, the electronic databases of Pubmed, Medline, Web of Science, Cochrane Embase, CBMand CNKI were systematically searched by two reviewers (Han Yanqing, Dong Cheng) independently. The publication searching terms were as follows: "colorectal cancer”, “colorectal carcinoma”, "colon cancer”, 
"rectal cancer", “CA72-4", "CA724", "Tumor-associated glycoprotein 72 (TAG-72)". The references of included studies were also screened to identify potential suitable publications.

\subsection{Study inclusion and exclusion criteria}

Inclusion criteria: (1) The patients were diagnosed with colorectal cancer with pathology conformation; (2)Serum level of CA72-4 was extracted from the original publications; (3)Enough data such as tpfpfn and tnwas extracted from the included studies to calculate the diagnostic sensitivity and specificity; (4) The language was limited to English and Chinese; Exclusion criteria: (1) Studies without enough data or cases, and use of abstract publications; (2) Colorectal cancer patients were not confirmed by pathology examination; (3) Duplicated publications or data; (4) Papers published in other languages other than English or Chinese.

\subsection{Quality assessment of the included studies}

The general methodological qualities of the 22 included publications were assessed by two reviewers (Dong Cheng and $\mathrm{Xu}$ Ling) independently through an eleven items questionnaire provided by Cochrane Reviews Handbook.

\subsection{Statistical method}

The statistical heterogeneity among the 22 included publications was evaluated by $\mathrm{I}^{2}$ test. If $\mathrm{I}^{2}>50 \%$, the data was pooled by Dersimonian-Laird method (random effect

Table 1: The main character of the included 22 publications

\begin{tabular}{|c|c|c|c|c|c|c|c|}
\hline Author & Year & tp & $\mathrm{fp}$ & fn & $\operatorname{tn}$ & Sensitivity & Specificity \\
\hline Fiorella & 1993 & 86 & 2 & 114 & 98 & $0.43(0.36-0.50)$ & $0.98(0.93-1.00)$ \\
\hline Sun Wei & 2000 & 21 & 2 & 37 & 27 & $0.36(0.24-0.50)$ & $0.93(0.77-0.99)$ \\
\hline He Hui & 2004 & 20 & 2 & 36 & 28 & $0.36(0.23-0.50)$ & $0.93(0.78-0.99)$ \\
\hline Yue Lin & 2006 & 30 & 3 & 57 & 57 & $0.34(0.25-0.45)$ & $0.95(0.86-0.99)$ \\
\hline Zhu Zili & 2006 & 45 & 15 & 22 & 63 & $0.67(0.55-0.78)$ & $0.81(0.70-0.89)$ \\
\hline Liu Shengli & 2007 & 34 & 2 & 57 & 33 & $0.37(0.27-0.48)$ & $0.94(0.81-0.99$ \\
\hline QiuBing & 2008 & 14 & 2 & 24 & 28 & $0.37(0.22-0.54)$ & $0.93(0.78-0.99)$ \\
\hline Dai Peng & 2008 & 55 & 14 & 85 & 19 & $0.39(0.31-0.48)$ & $0.58(0.39-0.75)$ \\
\hline Pan Aiping & 2009 & 56 & 13 & 29 & 65 & $0.66(0.55-0.78)$ & $0.83(0.73-0.91)$ \\
\hline Jiang Xiaoting & 2010 & 62 & 20 & 99 & 80 & $0.39(0.31-0.46)$ & $0.80(0.71-0.87)$ \\
\hline Huang Fujiao & 2011 & 14 & 3 & 27 & 43 & $0.34(0.20-0.51)$ & $0.93(0.82-0.99)$ \\
\hline Wan Caifeng & 2011 & 68 & 10 & 34 & 40 & $0.67(0.57-0.76)$ & $0.80(0.66-0.90)$ \\
\hline Yao Aiping & 2011 & 73 & 10 & 117 & 40 & $0.38(0.31-0.46)$ & $0.80(0.66-0.90)$ \\
\hline Hu Xiaoai & 2011 & 14 & 3 & 27 & 43 & $0.34(0.20-0.51)$ & $0.93(0.82-0.99)$ \\
\hline Chen Jun & 2012 & 292 & 7 & 62 & 46 & $0.82(0.78-0.86)$ & $0.87(0.75-0.95)$ \\
\hline Wang Xiuyin & 2012 & 64 & 34 & 48 & 182 & $0.57(0.47-0.66)$ & $0.84(0.79-0.89)$ \\
\hline Song Peidong & 2012 & 10 & 1 & 40 & 29 & $0.20(0.10-0.34)$ & $0.97(0.83-1.00)$ \\
\hline TianHua & 2013 & 35 & 27 & 25 & 93 & $0.58(0.45-0.71)$ & $0.78(0.69-0.85)$ \\
\hline Zhu Xiaofei & 2013 & 141 & 18 & 104 & 128 & $0.58(0.51-0.64)$ & $0.88(0.81-0.93)$ \\
\hline GuoQinhua & 2013 & 15 & 1 & 61 & 97 & $0.20(0.11-0.30)$ & $0.99(0.94-1.00)$ \\
\hline LvZhongchuan & 2013 & 63 & 21 & 97 & 79 & $0.39(0.32-0.47)$ & $0.79(0.70-0.87)$ \\
\hline Cheng Jinling & 2017 & 15 & 9 & 45 & 39 & $0.25(0.15-0.38)$ & $0.81(0.67-0.91)$ \\
\hline
\end{tabular}


model). Otherwise, the data was calculated by fixed-effect methods. The diagnostic performance of serum CA72-4 as a biomarker in the diagnosis of colorectal cancer was evaluated by sensitivity and specificity. [sensitivity=true positive/(true positive + false negative), specificity=true negative/( true negative+ false positive)]. All the data was analyzed by MetaDiSc 1.4 and Stata/SE 11.0 statistical software.

\section{Results}

\subsection{Main character of the included studies}

A total of 22 studies [6-27] with 2474 colorectal patients and 1576 controls were includedwith meta-analysis. Initially, 872 studies were identified through the electronic databases search. After reviewing the title, abstract and full text, 22 publications were finally included according to the study inclusion and exclusion criteria (Figure 1). The main character of the included 22 original studies is demonstrated by Table 1 .

\subsection{Methodological qualities of the included studies}

The methodological quality of the included 22 publications was evaluated by an 11 items questionnaire (Figure 2) and indicates the general quality was poor.

\subsection{Combined sensitivity for serum CA72-4 in diagnosis of colorectal cancer}

Due to statistical heterogeneity across the 22 studies $\left(I^{2}=93.7 \%\right)$, the data was pooled by Dersimonian-Laird method. The combined diagnostic sensitivity was 0.50 (95\%CI:0.48-0.52) for serum CA72-4 as a biomarker in the diagnosis of colorectal cancer, Figure 3.

\subsection{Combined specificity for serum CA72-4 in diagnosis of colorectal cancer}

The diagnostic specificity was pooled by random effect model because of statistical heterogeneity $\left(\mathrm{I}^{2}=78.2 \%\right)$. The pooled specificity was 0.86 (95\%CI:0.84-0.88) for serum CA72-4 as a biomarker in the diagnosis of colorectal cancer, Figure 4.

Figure 1: The publication searching flow chart

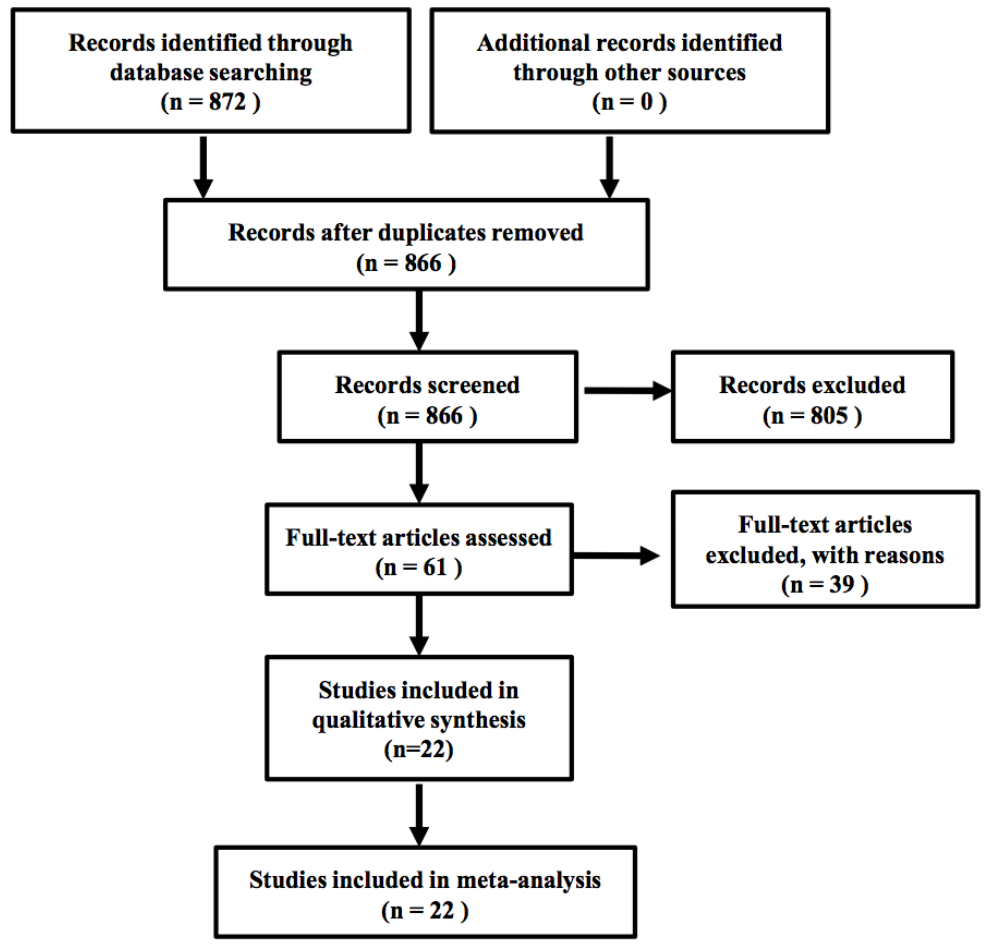




\subsection{Combined positive likelihoodratio (Positive LR)}

The data was pooled through random effect model because of significant statistical heterogeneity among the 22 publications $\left(\mathrm{I}^{2}=73.1 \%\right)$. The combined Positive LR was 3.41 (95\%CI:2.57-4.53), Figure 5.

\subsection{Combined negative likelihoodratio (negative LR)}

The negative LR was pooled by random effect model for significant statistical heterogeneity across the publications $\left(\mathrm{I}^{2}=89.5 \%\right)$. The combined negative $\mathrm{LR}$ was $0.62(0.55$ 0.71), Figure 6.

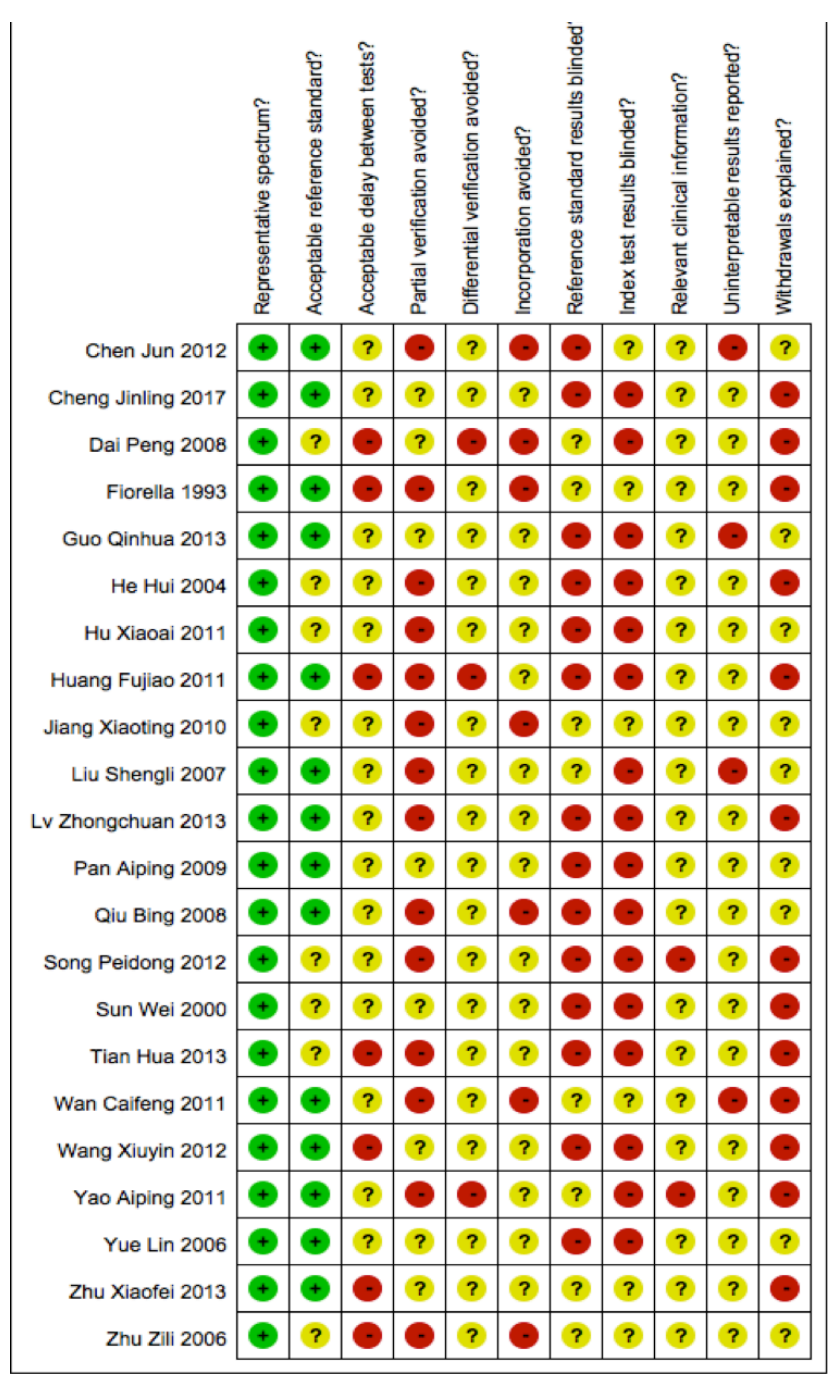

Figure 2: The methodological quality summary for each included study. (The authors'judgments for each risk of bias item. + is "low risk"; - is "high risk"; ?is"moderate risk").

\subsection{Combined diagnosed odds ratio(DOR)}

The diagnostic odds ratio (DOR) for serum CA72-4 as a biomarker in the diagnosis of colorectal cancer was pooled by random effects model. The pooled DOR was 6.18 with the $95 \%$ CI of 4.17-9.17, Figure 7.

\subsection{The diagnostic HSROC of serum CA72-4 in the diagnosis of colorectal cancer}

The pooled HSROC curve was calculated by sensitivity against (1-specificity). The pooled area under the ROC curve (AUC) was 0.73 for serum CA72-4 in the diagnosis of colorectal cancer, Figure 8.

\subsection{Publication bias analysis}

There was no publication bias of serum CA72-4 in the diagnosis of colorectal cancer assessed by Deeks'funnel plot (Figure 9) and Egger's line regression test $(\mathrm{p}=0.49)$.

\section{Discussion}

Colorectal cancer remains one of the most diagnosed malignant carcinomas that represents a global health problem [28]. It has previously been indicated that delayed diagnosis is the main reason for a poor prognosis. Over the past few decades, investigations have focused on the serum tumor markers for screening or early diagnosis of colorectal cancer. The most clinically used serum biomarkers for colorectal cancer screening or diagnosis in high risk population were CEA, CA199, CA125 CA72-4 and et al [29, 30].

Carbohydrate antigen 724(CA72-4), a specific glycoprotein antigen of the tumor associated glycoprotein (TAG-72), is initially used as serum biomarker for pancreatic cancer diagnosis. And recently, it was found that the serum CA72-4 level was elevated in patients with gastric cancer, ovarian cancer and colorectal cancer. Several studies [16-18] have evaluated its diagnostic performance as serum biomarkers for colorectal cancer. He et al [8] evaluated the serum levels of CA72-4 in diagnoses of colorectal cancer. They found that the serum concentration of CA72-4 was significantly higher in colorectal cancer patients compared to healthy controls. The serum level of CA72-4 was also positively correlated with the Duke's stage. Peidong $S$ evaluated the diagnostic performance of serum CA72-4 as 


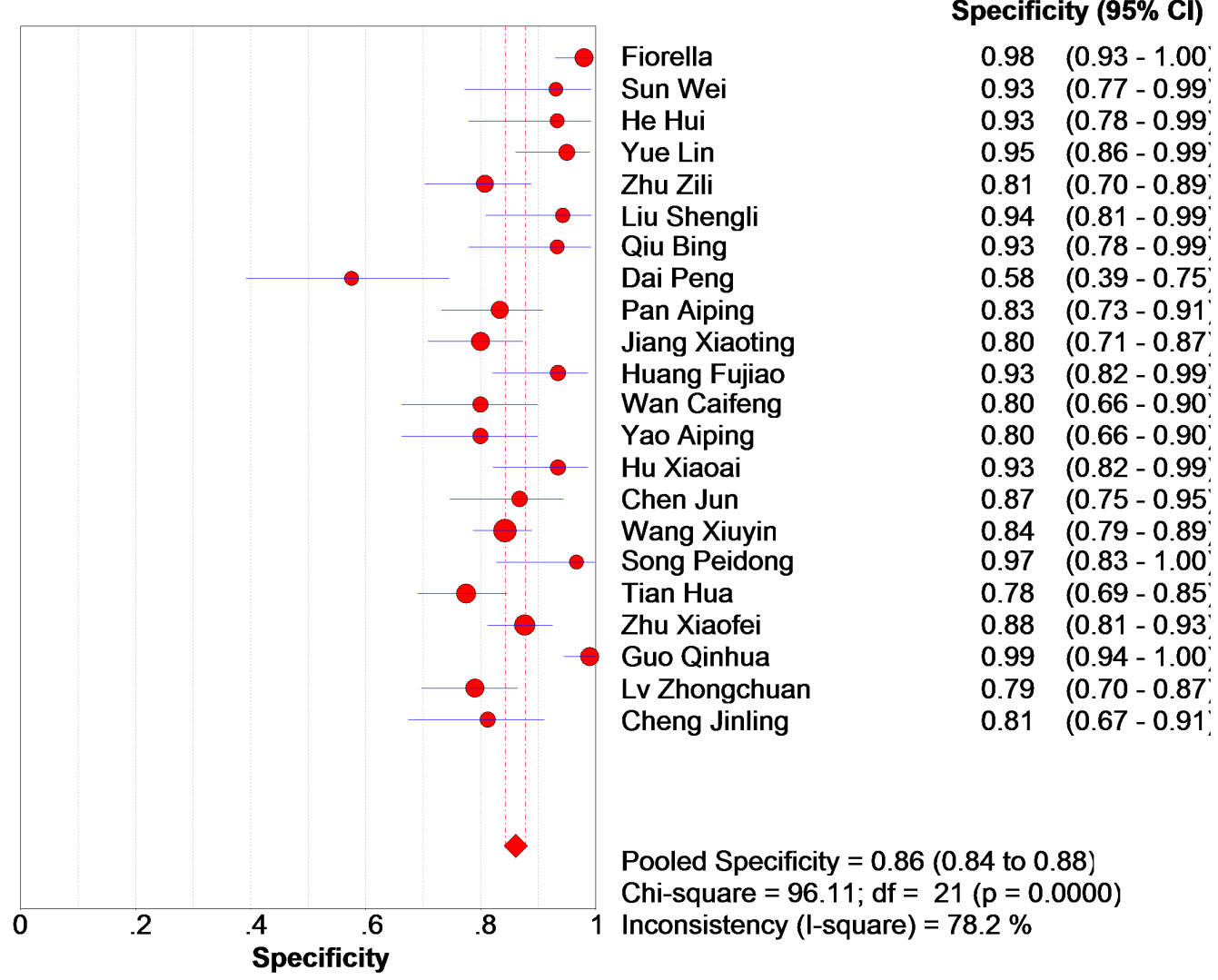

Figure 3: The diagnostic sensitivity of serum CA72-4 in the diagnosis of colorectal cancer

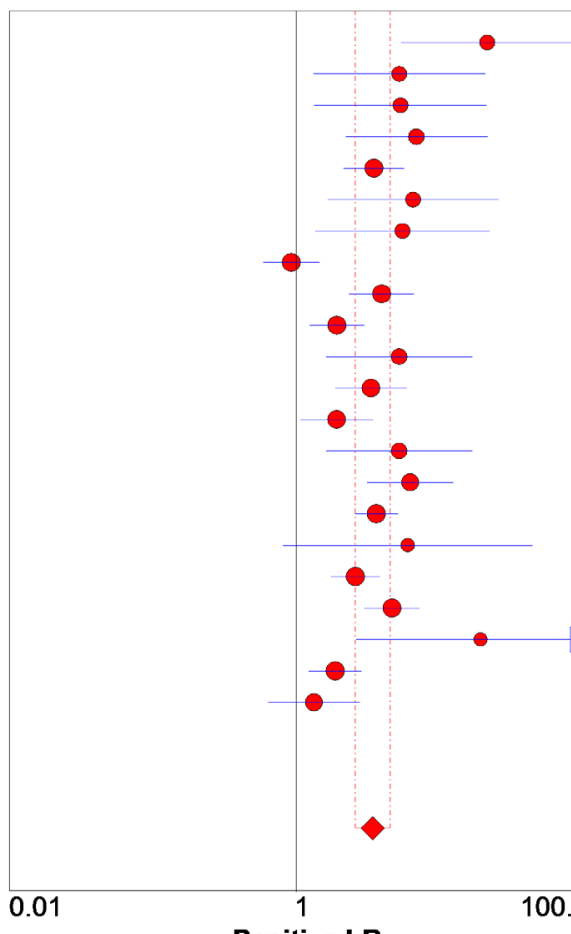

Positive LR
Fiorella

Sun Wei

He Hui

Yue Lin

Zhu Zili

Liu Shengli

Qiu Bing

Dai Peng

Pan Aiping

Jiang Xiaoting

Huang Fujiao

Wan Caifeng

Yao Aiping

Hu Xiaoai

Chen Jun

Wang Xiuyin

Song Peidong

Tian Hua

Zhu Xiaofei

Guo Qinhua

Lv Zhongchuan

Cheng Jinling
Positive LR (95\% Cl)

$21.50 \quad(5.40-85.57)$

$5.25 \quad(1.32-20.87)$

$5.36 \quad(1.34-21.38)$

$6.90 \quad(2.20-21.57)$

$3.49(2.15-5.67)$

$6.54 \quad(1.66-25.78)$

$5.53(1.36-22.46)$

$0.93 \quad(0.59-1.45)$

$3.95 \quad(2.35-6.64)$

$1.93(1.24-2.98)$

$5.24 \quad(1.62-16.93)$

$3.33(1.88-5.90)$

$1.92(1.07-3.44)$

$5.24(1.62-16.93)$

$6.25 \quad(3.13-12.47)$

$3.63(2.56-5.14)$

$6.00(0.81-44.56)$

$2.59 \quad(1.75-3.85)$

$4.67 \quad(2.99-7.29)$

$19.34 \quad(2.61-143.20$

$1.88(1.22-2.87)$

$1.33(0.64-2.78)$

Random Effects Model

Pooled Positive LR $=3.41$ (2.57 to 4.53 )

Cochran- $\mathrm{Q}=78.05 ; \mathrm{df}=21(\mathrm{p}=0.0000)$

Consistency (l-square) $=73.1 \%$

Tau-squared $=0.2753$

Figure 4: The diagnostic specificity of serum CA72-4 in the diagnosis of colorectal cancer 


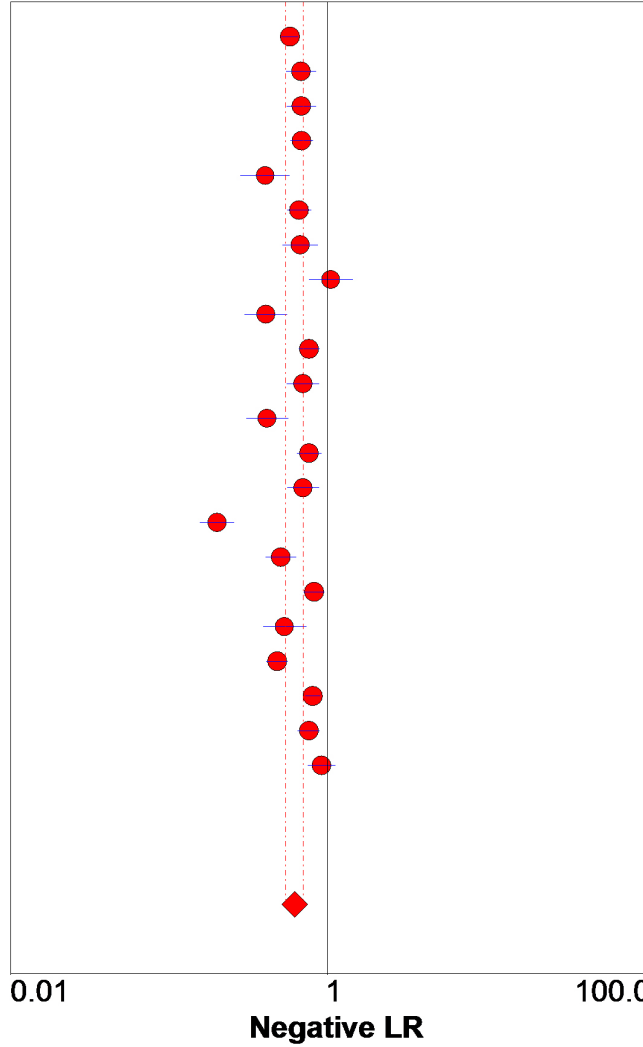

Fiorella

Sun Wei

He Hui

Yue Lin

Zhu Zili

Liu Shengli

Qiu Bing

Dai Peng

Pan Aiping

Jiang Xiaoting

Huang Fujiao

Wan Caifeng

Yao Aiping

Hu Xiaoai

Chen Jun

Wang Xiuyin

Song Peidong

Tian Hua

Zhu Xiaofei

Guo Qinhua

Lv Zhongchuan

Cheng Jinling
Negative LR (95\% C

$\begin{array}{ll}0.58 & (0.51-0.66 \\ 0.69 & (0.55-0.85 \\ 0.69 & (0.55-0.86 \\ 0.69 & (0.59-0.81 \\ 0.41 & (0.28-0.58 \\ 0.66 & (0.56-0.79 \\ 0.68 & (0.52-0.88 \\ 1.05 & (0.76-1.45 \\ 0.41 & (0.30-0.56 \\ 0.77 & (0.66-0.90 \\ 0.70 & (0.56-0.89 \\ 0.42 & (0.31-0.57 \\ 0.77 & (0.64-0.92 \\ 0.70 & (0.56-0.89 \\ 0.20 & (0.16-0.26 \\ 0.51 & (0.41-0.63 \\ 0.83 & (0.71-0.97 \\ 0.54 & (0.39-0.74 \\ 0.48 & (0.41-0.57 \\ 0.81 & (0.72-0.91 \\ 0.77 & (0.65-0.90 \\ 0.92 & (0.76-1.13\end{array}$

Random Effects Model

Pooled Negative LR $=0.62$ (0.55 to 0.71 )

Cochran-Q $=200.65 ; \mathrm{df}=21(p=0.0000)$

Inconsistency (I-square) $=89.5 \%$

Tau-squared $=0.0820$

Figure 5: Combined positive likelihoodratio(Positive LR) for serum CA72-4 in the diagnosis of colorectal cancer

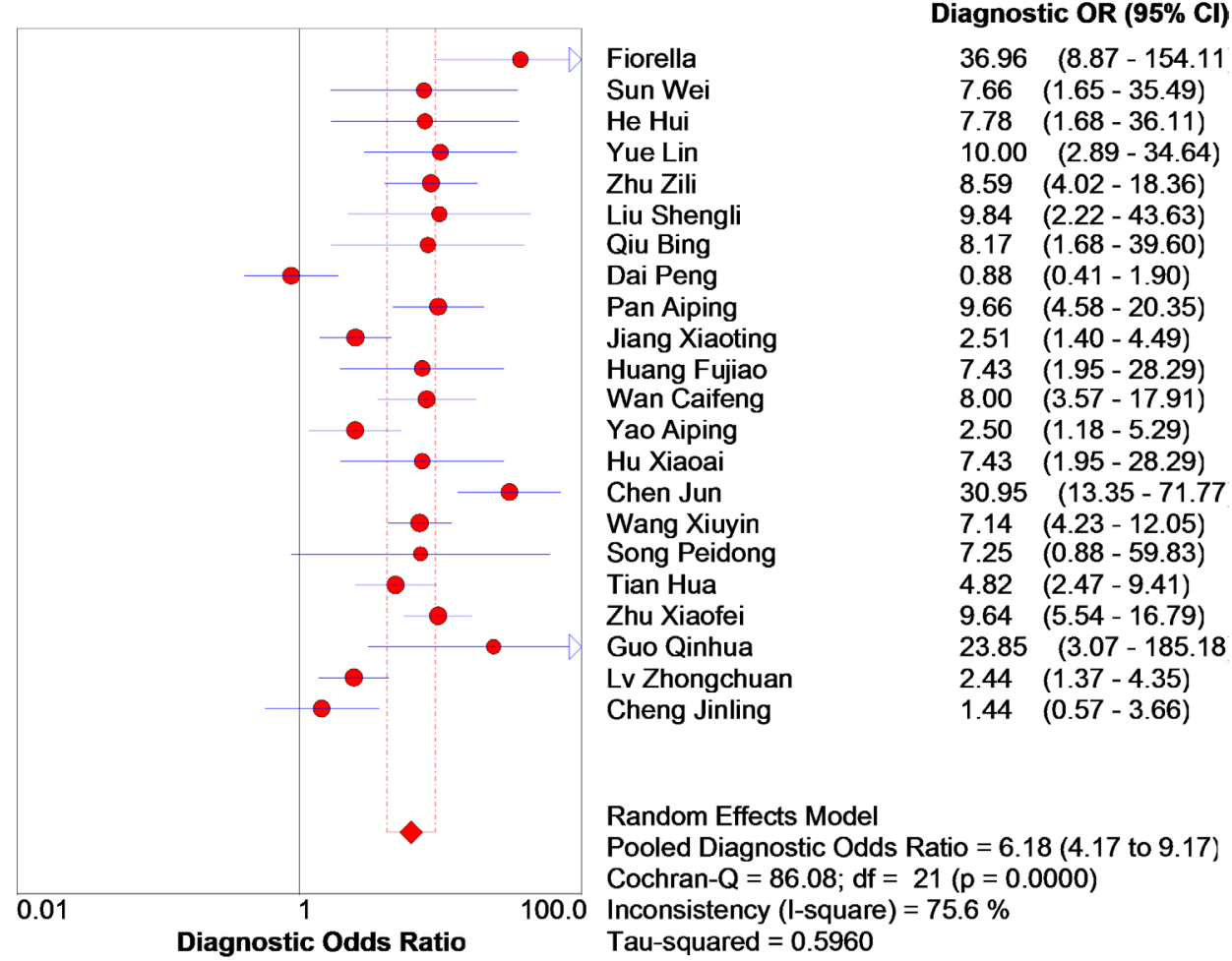

Figure 6: Combined negative likelihoodratio(negative LR) for serum CA72-4 in the diagnosis of colorectal cancer 


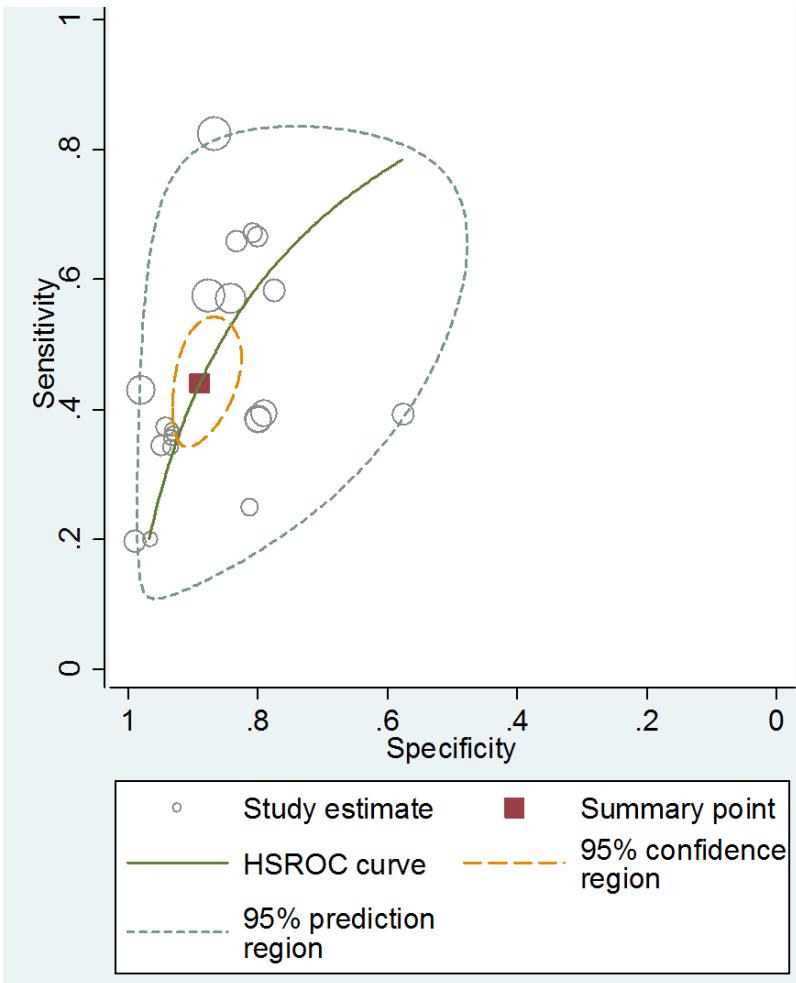

Figure 7: Combined DOR for serum CA72-4 in the diagnosis of colorectal cancer

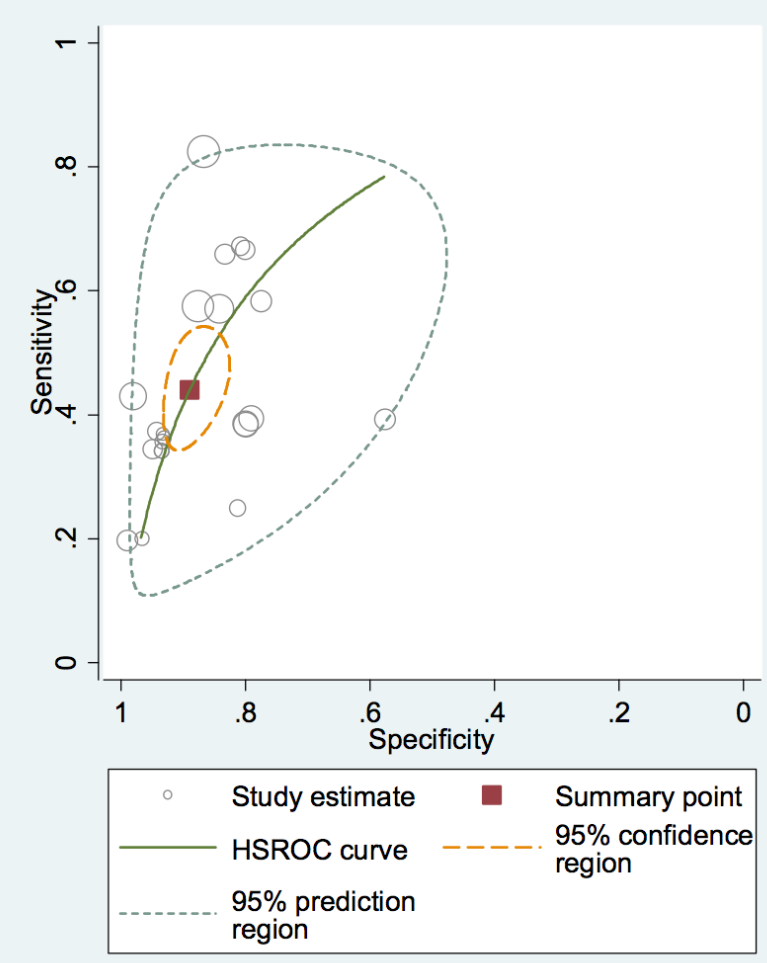

Figure 8: The pooled HSROC for serum CA72-4 in the diagnosis of colorectal cancer

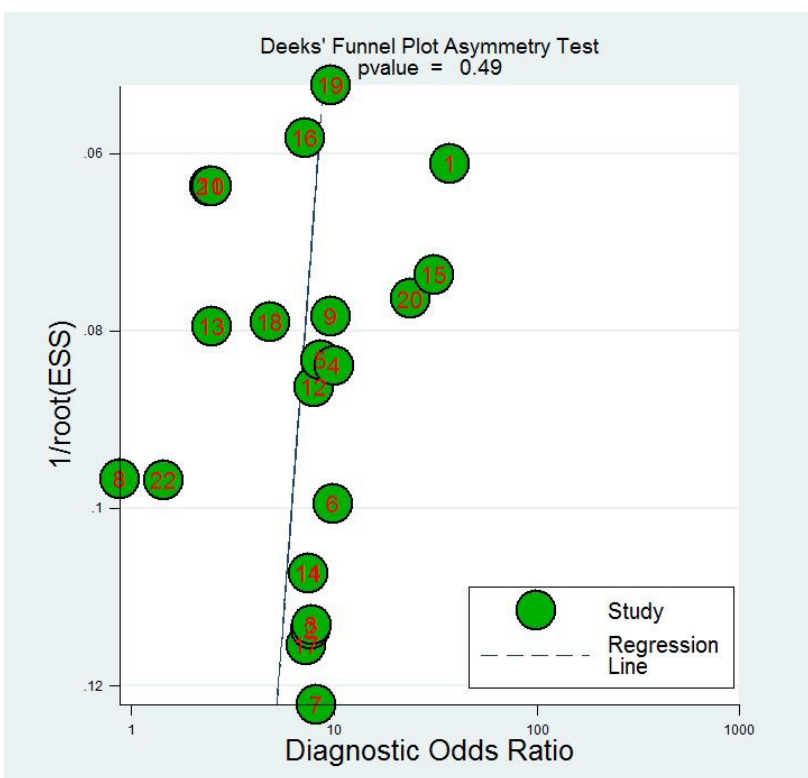

Figure 9: Deeks'funnel plot for evaluation of the publication bias

a biomarker for colorectal cancer diagnosis [22]. However, they found the diagnostic sensitivity of serum CA72-4 asunsuitable for colorectal cancer screening or diagnosis.

In our meta-analysis, we included 22 publications relevant to serum CA72-4 as a biomarker for colorectal cancer diagnosis and pooled the diagnostic sensitivity, specificity and hierarchical summary receiver operating characteristic curve (HSROC). The combined results demonstrated the diagnostic sensitivity and specificity as 0.50 (95\%CI:0.48-0.52) and 0.86 (95\%CI:0.84-0.88) respectively for serum CA72-4 as a biomarker in the diagnosis of colorectal cancer. The pooled area under the ROC curve (AUC) was 0.73 . The diagnostic performance was especially poor for sensitivity which shows serum CA72-4 is unsuitable as a biomarker for colorectal cancer screening. The low sensitivity for diagnosis of colorectal cancer always leads to high false negative which usually causes a high rate of missed diagnosis.

Several limitations were found in our present meta-analysis: (1) The general methodological quality of the included 22 studies was poor; (2) Significant heterogeneity was found across the 22 publications; (3) Only English and Chinese publications were searched and included in this meta-analysis.

Conflicts of interest: The authors have no conflicts of interest to declare. 


\section{References}

[1] Siegel RL, Miller KD, Jemal A. Cancer statistics, 2016. CA Cancer J Clin 2016;66:7-30

[2] Torre LA, Bray F, Siegel RL, Ferlay J, Lortet-Tieulent J, Jemal A. Global cancer statistics, 2012. CA Cancer J Clin 2015;65:87-108

[3] Fernandes LL, Martins LC, Nagashima CA, Nagae AC, Waisberg DR, Waisberg J. CA72-4 antigen levels in serum and peritoneal washing in gastric cancer. Correlation with morphological aspects of neoplasia.ArqGastroenterol 2007;44:235-239

[4] Granato T, Midulla C, Longo F, Colaprisca B, Frati L, Anastasi E. Role of HE4, CA72.4, and CA125 in monitoring ovarian cancer. TumourBiol 2012;33:1335-1339

[5] Chen C, Chen Q, Zhao Q, Liu M, Guo J. Value of Combined Detection of Serum CEA, CA72-4, CA19-9, CA15-3 and CA12-5 in the Diagnosis of Gastric Cancer. Ann Clin Lab Sci 2017; 47:260-263

[6] Guadagni F, RoselliM, Cosimelli M, Mannella E, Tedesco M, Cavaliere F, Grassi A, Abbolito MR, Greiner JW, Schlom J. TAG-72 (CA $72-4$ assay) as a complementary serum tumor antigen to carcinoembryonic antigen in monitoring patients with colorectal cancer. Cancer 1993;72:2098-2106

[7] Wei S, Qiong W, Juan L. The application of serum tumor markers in the diagnosis of colorectal cancer. CHINESE JOURNAL OF ONCOLOGY 2000;23:11-13

[8] Hui H, Dengqing L, Shaojie L, Wenkai L. Application of serum levels of CEA, CA19-9 and CA72-4 in diagnosing colorectal cancer. China Journal Of Modern Medicine 2004;14:79-80

[9] Zili Z, Jibin L, Jiangang L, Zhong C. Application of Combining Test of Serum Levels of Tumor Markers for the Clinical Diagnosis Value in the Colorectal Cancer. Journal Of Basic And Clinical Oncology 2006;19:469-470

[10] Lin Y, Guibin Y, Meihua C, Fanghong M. Clinical Usefulness of Serum Tumor markers CEA, CA19-9 and CA72-4 in Colorectal Cancer Diagnosis. Chinese Journal of Medicinal Guide 2006;8:346-347

[11] Lisheng L, Zuowei L, Ailan W, Yuhong L. The Clinical Application of CEA ,CA72-4 in Colorectal Cancer. Journal of Basic And Clinical Oncology 2007;20:43-44

[12] Bing Q, Ying L, Yanying W, Dongxia L. The application of combined detection of CEA, CA19-9 and CA72-4 in the diagnosis of gastric, colorectal cancer. Chinese Journal of Laboratory Diagnosis 2008;1017-1019

[13] Peng D. The significance of the determination of serum tumor markers in colorectal cancer[D].Shanxi University 2008

[14] Aiping P, Jian L, Juntao C. Diagnostic value of an artificial neural network model based on tumor markers for colorectal carcinoma. Journal Of Colorectal \& Anal Surgery 2009;15:380-382

[15] Xiao-ting J. Diagnostic Value of Combination Detection of Serum Tumor Markers for Colorectal Cancer. J Oncol 2010;16:97-99
[16] Xiaoai H, Guangbin C, Wei W. Application of combined determination of CEA and CA724 to the diagnosis of colorectal cancer. China Practical Medical 2011;06:54-55

[17] Wu-jiao H, Man LI, Zi-an WU, Ning XU, Xian-zhang H, Jun-hua Z, qian L. Combined Determination of CYFRA21-1, CEA and CA125 in Lung Cancers. Academic Journal Of Guangzhou Medical College 2011;27:2252-2254

[18] Aiping Y. Diagnostic Value of Combined Test of Serum Levels of Tumor Markers for Colo-reactal Cancer. Journal Of Radioimmunology 2011;24:78-80

[19] Caifeng W. The Diagnosis and Prognostic Value of the United Detection of TumorMarkers in the Patients with Cancer of Colon. Chinese Journal Of Clinical Oncology 2011;38:1169-1171

[20] Jun C. Diagnostic value of CA72-4, CA125, CA199 and CEA in gastric cancer, colorectal cancer and gastric stromal tumors. [D].Jilin University 2012

[21] Xiuyin W, Wenli C, Peilian L, Weiqing Z. Diagnostic value of combined detection of CEA, CA125 and CA724 for colorectal cancer. Guangzhou Medical Journal 2012;43:12-14

[22] Peidong S, Gencheng Z. The application of combined detection of serum tumor markers CEA, CA199, CA242 and CA724 in the diagnosis of colorectal cancer. Chinese Journal Of Laboratory Diagnosis 2012;16:1279-1281

[23] Hua T, Heming W, Jie C, Qiwen Y, Min L, Du Xiaozhen. Diagnostic value of combined detection of CEA, CA199, CA242 and CA724 for colorectal cancer. Modern Digestion \& Intervention 2013:317-318

[24] Lv ZC, Chen HB, Jiang LX, Hu XC, Wu GC, Zhao DW. The establishment and evaluation of the method of screening colorectal cancer with tumor markers. China Medical Engineering 2013:133-136

[25] Xiaofei Z. The value of combined detection of CA72-4, SF and CEA in colon cancer. Clinical Laboratory Journal 2013:352-354

[26] Qinhua G, Haiyan A, Qinghua C, Wanting Z, Fang H, Shuduan $X$. The value of combined examination of CEA, CA199 and CA72-4 in the diagnosis of colorectal cancer. International Journal of Laboratory Medicine 2013;34:896-897

[27] Jinling C. Diagnostic value of combined detection of CEA, CA19-9, CA242 and CA72-4 on rectal cancer. China Modern Doctor 2014:46-48

[28] Miller KD, Siegel RL, Lin CC, Mariotto AB, Kramer JL, Rowland JH, Stein KD, Alteri R, Jemal A. Cancer treatment and survivorship statistics, 2016. CA Cancer J Clin 2016;66:271-289

[29] Stojkovic LM, Stankovic S, Stojkovic M, Markovic V, Dimitrijevic I, Lalosevic J, Petrovic J, Brankovic M, Pavlovic MA, Krivokapic Z. Can preoperative CEA and CA19-9 serum concentrations suggest metastatic disease in colorectal cancer patients. Hell J Nucl Med 2017;20:41-45

[30] Nicholson BD, Shinkins B, Pathiraja I, Roberts NW, James TJ, Mallett S, Perera R, Primrose JN, Mant D. Blood CEA levels for detecting recurrent colorectal cancer. Cochrane Database Syst Rev 2015:CD011134 